\title{
Impact of Nocturia on Health-Related Quality of Life and Medical Outcomes Study Sleep Score in Men
}

\author{
Sun-Ouck Kim, Hyang Sik Choi, Yong Joong Kim, Hee Sun Kim, In Sang Hwang, Eu Chang Hwang, Kyung Jin Oh, \\ Seung Il Jung, Taek Won Kang, Dongdeuk Kwon, Kwangsung Park, Soo Bang Ryu \\ Department of Urology, Chonnam National University Medical School, Gwangju, Korea
}

\begin{abstract}
Purpose: To evaluate the impact of nocturia on health-related quality of life and sleep in men.
Methods: From January 2008 to December 2008, 284 patients with lower urinary tract symptoms were selected for this study. The participants completed a series of questionnaires on health-related quality of life (the overactive bladder questionnaire, or OAB-q), the Medical Outcomes Study (MOS) sleep scale, and the frequency volume chart.

Results: The patient population had a mean age of $60.0 \pm 13.4$ years (range, 40 to 79 years). The mean duration of symptoms was $28.8 \pm 34.6$ months. The mean number of voiding episodes per night was measured as follows: 88 patients (31.0\%) reported no nocturia, 60 patients (21.1\%) reported $2>$ voids/night $\geq 1$, 56 patients (19.7\%) reported $3>$ voids/night $\geq 2$, and 80 patients (28.2\%) reported $\geq 3$ voids/night. The mean number of nocturia episodes increased with age $(\mathrm{P}=0.001)$, and the number of nocturia episodes was significantly associated with the $\mathrm{OAB}-\mathrm{q}$ symptom score $(\mathrm{P}=0.001)$ and symptom bother $(\mathrm{P}=0.001)$. Among the categories of the MOS sleep scale, sleep index I $(\mathrm{P}=0.020)$, sleep disturbance $(\mathrm{P}=0.010)$, adequacy of sleep $(\mathrm{P}=0.005)$, and somnolence $(\mathrm{P}=0.041)$ were significantly associated with an increased number of nocturia episodes.

Conclusions: The number of nocturia episodes increased with age in men. Nocturia appeared to be associated with further negative effects on sleep quality, health-related quality of life, and symptom bother.
\end{abstract}

Keywords: Nocturia; Sleep; Quality of life

\section{INTRODUCTION}

Nocturia, one of the most prevalent urinary symptoms in adults, is regarded as having a significant impact on sleep and quality of life [1]. According to the definition of the International Continence Society, nocturia is the complaint that an individual wakes one or more times to void urine at night [2]. In a nationwide Asian epidemiologic study, Homma et al. [3] reported that nighttime frequency of voiding was the most bothersome symptom among various lower urinary tract symptoms (LUTS).

Asplund and Aberg [4] also reported that nocturia can cause daytime fatigue, decreased general well-being, and increased risk of nighttime falling. Fragmented and disturbed sleep patterns may be observed in patients with nocturia that can result in disturbance of good sleep and daytime sleepiness and result in serious health risks in this population [5]. Middelkoop et al. [6] suggested that nocturia is a main factor of disturbance of sleep maintenance in adults aged 50 years or older. Su et al. [7] reported that nocturia increases the risk of insomnia 20.6-fold. Therefore, it is important to determine how much of an impact nocturia has on sleep quality and health-related quality of life and how they are correlated with each other. The aim of the present study was therefore to evaluate the effects of nocturia on sleep and health-related quality of life.
Corresponding author: Dongdeuk Kwon

Department of Urology, Chonnam National University Hospital, Chonnam National University Medical School, 8 Hak-dong, Dong-gu, Gwangju 501-757, Korea

Tel: +82-62-220-6705 / Fax: +82-62-227-1643 / E-mail: urokwon@gmail.com Submitted: June 1, 2011 / Accepted after revision: June 21, 2011
This is an Open Access article distributed under the terms of the Creative Commons Attribution Non-Commercial License (http://creativecommons.org/licenses/by-nc/3.0/) which permits unrestricted non-commercial use, distribution, and reproduction in any medium, provided the original work is properly cited. 


\section{MATERIALS AND METHODS}

\section{Participants}

This study was conducted from January 2008 to December 2008 among patients who visited our outpatient clinic with LUTS. All patients underwent a detailed clinical evaluation involving a complete history taking, a physical examination, a digital rectal examination, completion of the International Prostate Symptom Score (IPSS), urinalysis, and measurement of prostate-specific antigen. A total of 284 subjects who were aged $>40$ years, had moderate to severe LUTS (IPSS sum $>8$ ), and postvoid residual urine $<100 \mathrm{~mL}$ were included. Participants completed a series of questionnaires on health-related quality of life (validated Korean version of the overactive bladder questionnaire, or OABq), the Medical Outcomes Study (MOS) sleep scale, and a frequency volume (FV) chart.

Subjects with a confused or depressed mental status, taking medication such as sedatives or tranquillizers, taking medications that may alter or control bladder symptoms and sleep quality, with a history of a previous surgery for LUTS, with a history of urinary retention, or with symptomatic urinary tract infection, uropathologic conditions such as urinary stones and urogenital cancer, or neurogenic bladder were not included. Patients with symptoms of urinary incontinence or restricted mobility and patients who worked at night were also excluded from this analysis. All patients signed an informed consent agreement.

\section{Frequency Volume Chart}

Patients were asked to complete 3-day FV charts. They were taught how to precisely complete the FV charts by the urologist and were asked not to alter their usual fluid intake and voiding habits during the study. The times that they arose in the morning and went to bed at night were recorded on the FV charts. Numbers and volumes of voids were estimated by taking a mean over the 3-day study period. Nighttime was defined as the time from bedtime to rising in the morning.

\section{Overactive Bladder Questionnaire}

The OAB-q is a 33-item self-administered symptom bother scale that assesses the patient's current bladder condition; the first 8 items assess symptom bother and the remaining 25 items assess health-related quality of life. Domains include coping, concern, sleep, and social interaction [8].

\section{Medical Outcome Study Sleep Scale}

The MOS sleep scale yields a sleep problems index and six scale scores: sleep disturbance (have trouble falling asleep, how long to fall asleep, sleep was not quiet, awaken during your sleep time, and have trouble falling asleep again), sleep adequacy (get enough sleep to feel rested upon waking in the morning, get the amount of sleep needed), daytime somnolence (drowsy during the day, have trouble staying awake during the day, take naps), snoring, awaken with shortness of breath or with headache, and quantity of sleep [9]. Quantity of sleep is scored as the average number of hours slept per night. The other scales and problems index are scored on a possible range of from 0 to 100, and higher scores indicate more of the concept being measured.

\section{Statistical Analysis}

For statistical analysis, patients were stratified by age groups and number of nocturia episodes. Data were compared statistically by using analysis of variance. A $5 \%$ level of significance was established for all of the statistical testing. Statistical analysis was performed by using SPSS ver. 13.0 (SPSS Inc., Chicago, IL, USA).

\section{RESULTS}

Patient characteristics are listed in Table 1 . The patient population had a mean age of $60.0 \pm 13.4$ years (range, 40 to 79 years). The mean duration of symptoms was $28.8 \pm 34.6$ months. The incidences of hypertension and cardiovascular disease were

Table 1. Patient characteristics and comorbidities

\begin{tabular}{lc}
\hline Characteristics & Value \\
\hline No. of patients & 284 \\
Mean age $(\mathrm{yr})$ & $60.0 \pm 13.4$ \\
Duration of symptom $(\mathrm{mo})$ & $28.8 \pm 34.6$ \\
Additional combinded disease & \\
Hypertention & $70(24.6)$ \\
Diabetus mellitus & $52(18.3)$ \\
Cardiovascular disease & $20(7.0)$ \\
Others & $6(2.1)$ \\
No. of nocturia & \\
Nocturia $<1$ & $88(31.0)$ \\
$2>$ Nocturia $\geq 1$ & $60(21.1)$ \\
$3>$ Nocturia $\geq 2$ & $56(19.7)$ \\
Nocturia $\geq 3$ & $80(28.2)$ \\
\hline
\end{tabular}

Values are presented as mean \pm SD or number (\%). 
$24.6 \%$ and $18.3 \%$, respectively (Table 1 ). The mean number of voiding episodes per night was measured as follows: 88 patients (31.0\%) reported no nocturia, 60 patients $(21.1 \%)$ reported $2>$ voids/night $\geq 1,56$ patients (19.7\%) reported $3>$ voids/night $\geq 2$, and 80 patients (28.2\%) reported $\geq 3$ voids/night (Table 1 ).

The mean number of nocturia episodes increased with age $(\mathrm{P}=0.001$, Table 2$)$. The number of nocturia episodes was significantly associated with the $\mathrm{OAB}-\mathrm{q}$ symptom score $(\mathrm{P}=0.001)$ and symptom bother $(\mathrm{P}=0.001$, Table 3$)$. Among the categories of the MOS sleep scale, sleep index $1(\mathrm{P}=0.020)$, sleep disturbance $(\mathrm{P}=0.010)$, adequacy of sleep $(\mathrm{P}=0.005)$, and somnolence $(P=0.041)$ were significantly associated with an increased number of nocturia episodes (Table 4).

Table 2. Age related classification of nocturia

\begin{tabular}{lccc}
\hline Age (yr) & No. of patients (\%) & $\begin{array}{c}\text { No. of nocturia } \\
(\text { mean } \pm \text { SD) }\end{array}$ & P-value \\
\hline $40-49$ & $60(21.1)$ & $0.96 \pm 1.22$ & 0.001 \\
$50-59$ & $67(23.6)$ & $1.31 \pm 2.00$ & \\
$60-69$ & $105(37.0)$ & $2.24 \pm 1.34$ & \\
$\geq 70$ & $52(18.3)$ & $2.63 \pm 2.07$ & \\
\hline
\end{tabular}

P-values calculated with analysis of variance.

\section{DISCUSSION}

In the present study, we evaluated the impact of nocturia on health-related quality of life and quality of sleep in men. Overall, we found that the prevalence of nocturia increased with age. Furthermore, nocturia was significantly associated with a decreased quality of life symptom score and symptom bother on the OAB-q and decreased sleep quality on the MOS sleep scale. Among the several categories of the MOS sleep scale, sleep index I, sleep disturbance, adequacy of sleep, and somnolence were significantly associated with an increased number of nocturia episodes. Given the impact of nocturia on these health-related quality of life indexes and sleep, nocturia should be routinely assessed by urologic clinicians and should be treated with consideration of its impact on these symptoms.

According to the definition of the International Continence Society, nocturia is the complaint that an individual has to awaken at night one or more times to void urine [2]. Nocturia has been recognized as a discrete clinical condition rather than a simple symptom. Nocturia has a negative impact on quality of life, affecting both morbidity and mortality. Many studies have examined the influence of nocturia on well-being and quality of life and have shown a consistently high impact of nocturia

Table 3. Quality of life and symptom bother according to nocturia

\begin{tabular}{lccccc}
\hline & No nocturia & $2>$ Nocturia $\geq 1$ & 3 $>$ Nocturia $\geq 2$ & Nocturia $\geq 3$ & P-value \\
\hline Mean age & $53.6 \pm 14.8$ & $55.6 \pm 14.3$ & $62.3 \pm 10.3$ & $67.0 \pm 9.4$ & 0.001 \\
HRQoL total & $85.5 \pm 12.6$ & $83.0 \pm 13.3$ & $76.4 \pm 22.4$ & $65.2 \pm 22.6$ & 0.001 \\
Symptom bother & $23.8 \pm 20.7$ & $25.2 \pm 21.9$ & $30.8 \pm 23.8$ & $46.2 \pm 27.5$ & 0.001
\end{tabular}

HRQoL, health related Quality of Life.

P-values calculated with analysis of variance.

Table 4. Sleep quality according to nocturia

\begin{tabular}{lccccc}
\hline MOS sleep scale & No nocturia & $2>$ nocturia $\geq 1$ & $3>$ nocturia $\geq 2$ & Nocturia $\geq 3$ & P-value \\
\hline Sleep index I & $31.5 \pm 16.2$ & $33.9 \pm 17.3$ & $36.9 \pm 17.0$ & $43.1 \pm 17.1$ & 0.020 \\
Hours sleep/night & $6.1 \pm 1.4$ & $6.7 \pm 1.4$ & $6.3 \pm 1.6$ & $6.2 \pm 1.6$ & 0.519 \\
Sleep disturbance & $29.9 \pm 18.5$ & $31.6 \pm 22.4$ & $33.5 \pm 22.2$ & $45.8 \pm 20.5$ & 0.010 \\
Snoring & $38.8 \pm 28.7$ & $39.3 \pm 24.9$ & $37.9 \pm 27.9$ & $44.5 \pm 26.6$ & 0.723 \\
Shortness of breath & $19.3 \pm 23.2$ & $20.6 \pm 27.5$ & $23.6 \pm 26.7$ & $26.5 \pm 26.9$ & 0.661 \\
Adequacy of sleep & $39.7 \pm 20.5$ & $42.6 \pm 20.8$ & $45.5 \pm 23.5$ & $48.5 \pm 22.5$ & 0.005 \\
Somnolence & $36.0 \pm 16.1$ & $39.2 \pm 21.4$ & $42.4 \pm 21.9$ & $44.5 \pm 18.5$ & 0.041 \\
\hline
\end{tabular}

MOS, Medical Outcomes Study.

$\mathrm{P}$-values calculated with analysis of variance. 
on these variables [10]. Nocturia has also been associated with an increased risk of falling down, limb fracture, excessive daytime somnolence, and nocturnal enuresis, when nighttime wakening occurs two or more times, with an increased risk of from 10 to $21 \%$ [11]. The reported impairment of quality of life is similar to that of type II diabetes patients complaining of nocturia twice or more at night [12]. General well-being is associated with fewer nighttime voids and better sleep quality, and days absent from work in working women due to sickness is related to the frequency of nighttime voiding [4].

Quality of life is negatively affected in most individuals who experience nocturia; this is perhaps to be expected of a condition that disrupts healthy sleep patterns. Sleep is essential to all biological individuals and is a phenomenon of a physiologic state of unconsciousness and inactivity of the voluntary muscles [13]. It is suggested that unfragmented sleep, 7 to 8 hours for most adults, is necessary for maintenance of physical, mental, and emotional well-being [14]. The abnormal sleep patterns observed in patients with nocturia can result in sleep deprivation and daytime sleepiness, lack of concentration and coordination, reduction in creativity, and alteration of mood, which may lead to serious health risks in the general population $[5,15]$. As a consequence, nocturia may have clinical implications regarding daytime activity and general health status. Sleep disturbance caused by nocturnal urinary frequency may result in decreased cognitive function during daytime periods, decreased performance at work, and depression [16]. A link between sleep problems and depression in older men has been observed with a stronger relationship noted in association with increasing nocturnal frequency [17]. Recently, there has also been some evidence to suggest that sleep quality has an impact on general immune system and host defense mechanisms, and, thereby, that sleep disturbance is associated with increased morbidity and mortality $[4,18,19]$.

In the typical adult, symptoms of nocturia are considered to be one of the most common reasons for interrupted sleep. Middlekoop et al. [6], who examined the way in which nocturia symptoms impacted sleep quality in a Dutch cross-sectional epidemiologic study, reported that nocturia and worry were the most important causes of sleep disturbance in adults over 50 years of age. The effects of nocturia on sleep disturbance have also been investigated in an elderly Swedish population. A close link was found between nocturia and sleep quality; nocturia was associated with an increased prevalence of sleep disorders, poorer quality of sleep, and increased daytime fatigue [4]. More recently, Yoshimura et al. [20] conducted a community-based study to determine the relationships among nocturia, sleep disturbance, and general health-related quality of life. They confirmed that an elevated nighttime frequency markedly reduces most aspects of health-related quality of life. They also observed that nighttime frequency had a close association with many aspects of sleep problems, among other factors, including chronic disease.

The prevalence of nocturia can increase in a linear fashion in association with increasing age, and the bother associated with nocturia symptoms also increases [20,21]. In the present study, we found consistent results showing an increased mean number of nocturia episodes with increased age. In addition to the age-related physiological changes to lower urinary tract function, nocturia in the elderly appears to result in a higher daily urine production than that in age-matched controls without nocturia. There has been a suggestion that these physiological changes might lead to an older adult experiencing an increased nocturnal urinary frequency [22] and a reduction in functional bladder capacity and reduced sensation of bladder filling. Many older people tend to have drinking habits that include fluid intake late at night, such as alcohol [23]. Diabetes mellitus and heart failure can induce polyuria due to raised arterial natriuretic peptide levels and an increased drive to thirst. Hypercalcemia resulting from oral calcium supplementation, dependent edema due to venous stasis or hypoalbuminemia, and diuretic medication late in the day causing edema have also been suggested as etiologies of nocturia in the elderly population [24]. Nocturia has also been associated with obstructive sleep apnea, related to elevated nighttime urine production and atrial natriuretic peptide excretion in response to cardiac distension caused by negative pressure within the chest. Therefore, a patient's usual pattern of fluid intake and drinking habits, such as alcohol consumption, should be ascertained. A detailed drug history, such as diuretics and antihypertensive medication, should be taken and counsel should to provided to make alterations if possible. Understanding the etiology of nocturia is important for appropriate management; however, it can have a complex and multifactorial origin.

There are several limitations to this study. The sample size was small and young adults were not included. This study was a cross-sectional study and is subject to recall bias. Many important variables in this analysis, such as comorbid conditions and medications that can affect the condition of the bladder and nocturia, were based entirely on participant reports without 
clinical verification. We used the FV chart for 3 days. Also, we were not able to check the amount of water drunk by our patients, who recorded voided volume and the time of each micturition, day and night. Despite these limitations, we believe that our study provides some valuable clues for treatment of patients with nocturia, with significant consideration for associated sleep disturbance.

In conclusion, the number of nocturia episodes increased with age in men. Nocturia appeared to be associated with further negative effects on sleep quality, health-related quality of life, and symptom bother.

\section{CONFLICT OF INTEREST}

No potential conflict of interest relevant to this article was reported.

\section{REFERENCES}

1. Lose G, Alling-Møller L, Jennum P. Nocturia in women. Am J Obstet Gynecol 2001;185:514-21.

2. van Kerrebroeck P, Abrams P, Chaikin D, Donovan J, Fonda D, Jackson S, et al. The standardisation of terminology in nocturia: report from the Standardisation Sub-committee of the International Continence Society. Neurourol Urodyn 2002;21:179-83.

3. Homma Y, Kakizaki H, Gotoh M, Takei M, Yamanishi T, Hayashi K. Epidemiologic survey on lower urinary tract symptoms in Japan. Nippon Hainyokino Gakkaishi 2003;14:266-77.

4. Asplund R, Aberg H. Health of the elderly with regard to sleep and nocturnal micturition. Scand J Prim Health Care 1992;10:98-104.

5. Weiss JP, Blaivas JG. Nocturia. J Urol 2000;163:5-12.

6. Middelkoop HA, Smilde-van den Doel DA, Neven AK, Kamphuisen HA, Springer CP. Subjective sleep characteristics of 1,485 males and females aged 50-93: effects of sex and age, and factors related to self-evaluated quality of sleep. J Gerontol A Biol Sci Med Sci 1996; 51:M108-15.

7. Su TP, Huang SR, Chou P. Prevalence and risk factors of insomnia in community-dwelling Chinese elderly: a Taiwanese urban area survey. Aust N Z J Psychiatry 2004;38:706-13.

8. Coyne KS, Matza LS, Thompson CL. The responsiveness of the Overactive Bladder Questionnaire (OAB-q). Qual Life Res 2005;14:
849-55.

9. Hays RD, Stewart AL. Sleep measures. In: Stewart AL, Ware JE, editors. Measuring functioning and well-being: the medical outcomes study approach. Durham: Duke University Press; 1992. p. 235-59.

10. Miceli P. The prevalence of nocturia and its effect on health-related quality of life and sleep in a community sample in the USA. BJU Int 2004;94:194-5.

11. Barker JC, Mitteness LS. Nocturia in the elderly. Gerontologist 1988; 28:99-104.

12. Coyne KS, Zhou Z, Bhattacharyya SK, Thompson CL, Dhawan R, Versi E. The prevalence of nocturia and its effect on health-related quality of life and sleep in a community sample in the USA. BJU Int 2003;92:948-54.

13. Tobler I. Why do we sleep? Contributions from animal research. Ther Umsch 2000;57:417-20.

14. Marschall-Kehrel D. Update on nocturia: the best of rest is sleep. Urology 2004;64(6 Suppl 1):21-4.

15. Broman JE, Lundh LG, Hetta J. Insufficient sleep in the general population. Neurophysiol Clin 1996;26:30-9.

16. Hetta J. The impact of sleep deprivation caused by nocturia. BJU Int 1999;84 Suppl 1:27-8.

17. Asplund R, Henriksson S, Johansson S, Isacsson G. Nocturia and depression. BJU Int 2004;93:1253-6.

18. Benca RM, Quintas J. Sleep and host defenses: a review. Sleep 1997; 20:1027-37.

19. Irwin M, McClintick J, Costlow C, Fortner M, White J, Gillin JC. Partial night sleep deprivation reduces natural killer and cellular immune responses in humans. FASEB J 1996;10:643-53.

20. Yoshimura K, Terada N, Matsui Y, Terai A, Kinukawa N, Arai Y. Prevalence of and risk factors for nocturia: analysis of a health screening program. Int J Urol 2004;11:282-7.

21. Kim SO, Kim JS, Kim HS, Hwang EC, Oh KJ, Kwon D, et al. Age related change of nocturia in women. Int Neurourol J 2010;14:245-9.

22. Matthiesen TB, Rittig S, Nørgaard JP, Pedersen EB, Djurhuus JC. Nocturnal polyuria and natriuresis in male patients with nocturia and lower urinary tract symptoms. J Urol 1996;156:1292-9.

23. Mitteness LS. The management of urinary incontinence by community-living elderly. Gerontologist 1987;27:185-93.

24. Wagg A, Andersson KE, Cardozo L, Chapple C, Kirby M, Kelleher C, et al. Nocturia: morbidity and management in adults. Int J Clin Pract 2005;59:938-45. 